\title{
GC 2012-5594: EXTERNALLY FUNDED POSTDOCS AND INTERNSHIP OPPORTUNITIES @ HP LABS
}

\author{
Mrs. Lueny Morell, Hewlett-Packard Corporation
}

Lueny Morell, MS, PE, is Program Manager in the Strategy Team of Hewlett Packard Laboratories (HPL) in Palo Alto, California. She focuses on identifying Open Innovation opportunities for HP Labs, emphasis on engineering/technology talent programs funded by external organizations. Since joining HP Labs in 2002 she has lead the development of various strategic partnerships with HP partners (governments, customers and universities) to catalyze ideas, resources and develop talent for innovation. Lueny also has developed engineering/science curriculum innovation initiatives worldwide in support of HPL research and technology areas and from 2002 to 2006 was the director of HPL University Relations for Latin America and the Caribbean in charge of building research and education collaborations with universities throughout the region. Before joining HP, Lueny was full professor of Chemical Engineering at the University of Puerto Rico Mayagez Campus where she held various positions including associate dean of engineering and director Campus R\&D. Recipient of the 2006 US National Academy of Engineering Bernard M. Gordon award for innovations in the engineering curriculum and the 2010 Latin America and the Caribbean Consortium of Engineering Institutions Academic Award, her work in curriculum, research, accreditation and economic development activities has been published in more than 85 papers, book chapters and journals. She is a licensed engineer, an IEEE Senior Member, an ASEE Fellow, an ABET reviewer and member of various national and international boards including the US National Academies Board of International Science Organizations, the US National Science Foundation International Science and Engineering Advisory Committee, Past President of the International Federation of Engineering Education Societies, engineering expert of the OECD AHELO project and Co-Director of the International Institute for Developing Engineering Academics (IIDEA). Together with colleagues, Lueny has offered more than 90 engineering education, curriculum/learning environments innovation workshops around the world. 


\title{
Externally Funded Postdocs and Internship Opportunities @ HP Labs
}

\author{
Submitted by \\ Lueny Morell, Program Manager, Strategy Team, Hewlett Packard Laboratories \\ lueny.morell@hp.com
}

Innovation and invention represent the livelihood of companies in a flat world. Companies must innovate or perish. Both advances in technology, and the entrepreneurial culture that is well ingrained in the mindset of successful companies and their research labs, need to find a way into science and engineering higher education systems to help to develop talent that can not only use technology, but also help create it and develop new business opportunities with it. Universities are usually the preferred venue for post-doctoral candidates (postdocs) to spend 2 to 3 years further expanding their research interests, experience and networks before formally initiating their careers with a company, university, or self-owned business. Nevertheless, industry research labs can also provide ways to engage not only faculty and students but also postdocs in innovation and in the transfer of state-of-the-art research results and emerging technology areas into new businesses. In corporate labs future science and technology leaders can experience real-life R\&D and entrepreneurial experiences that turn inventions into products and services. In other words, they can experience research with a purpose, other than publishing papers or writing theses. While Hewlett Packard Laboratories (HP Labs) has a long tradition of hosting postdocs and interns (e.g., in 2011 HP Labs hosted well over 100 interns at its Palo Alto facility), during the last 3 years it has been providing research experiences to postdocs, MS/PhD students and recently graduated engineers who are funded by government agencies. This presentation will provide a brief description of HP Labs research portfolio followed by a few examples of intern and postdoc experiences. 